Editorial

\title{
Recent Advances on Optimization for Control, Observation, and Safety
}

\author{
Guillermo Valencia-Palomo $1, * \mathbb{(})$, Francisco-Ronay López-Estrada $2, *$ (i) \\ and Damiano Rotondo ${ }^{3,4, *(D)}$ \\ 1 Tecnológico Nacional de México/Instituto Tecnológico de Hermosillo, Av. Tecnológico y Periférico Poniente, \\ S/N, Hermosillo 83170, Sonora, Mexico \\ 2 Tecnológico Nacional de México/Instituto Tecnológico de Tuxtla Gutiérrez. TURIX-Dynamics Diagnosis and \\ Control Group, Carretera Panamericana km 1080, Tuxtla Gutierrez C.P. 29050, Mexico \\ 3 Institut de Robòtica i Informàtica Industrial, CSIC-UPC, Llorens i Artigas 4-6, 08028 Barcelona, Spain \\ 4 Research Center for Supervision, Safety and Automatic Control, Universitat Politècnica de Catalunya (UPC), \\ Rambla Sant Nebridi, 22, 08022 Terrassa, Spain \\ * Correspondence: gvalencia@ith.mx (G.V.-P.); frlopez@ittg.edu.mx (F.-R.L.-E.); \\ damiano.rotondo@upc.edu (D.R.)
}

Received: 28 January 2020; Accepted: 31 January 2020; Published: 6 February 2020

\section{Introduction}

Mathematical optimization is the selection of the best element in a set with respect to a given criterion. Optimization has become one of the most commonly used tools in modern control theory to compute control laws, adjust the controller parameters (tuning), estimate unmeasured states, find suitable conditions to fulfill a given closed-loop property, carry out model fitting, among others. Optimization is also used in the design of fault detection and isolation systems, due to the complexity of automated installations and to prevent safety hazards and huge production losses that require the detection and identification of any kind of fault, as early as possible, as well as the minimization of their impacts by implementing real-time fault detection and fault-tolerant operations systems where optimization algorithms play an important role. Recently, it has been proved that many optimization problems with convex objective functions and linear matrix inequality (LMI) constraints can be solved efficiently using existing software, which increases the flexibility and applicability of the control algorithms. Therefore, real-world control systems need to comply with several conditions and constraints that have to be taken into account in the problem formulation, which represents a challenge in the application of the optimization algorithms.

This special issue aims at offering an overview of the state-of-the-art of the most advanced (online and offline) optimization techniques and their applications in control engineering.

\section{Papers Presented in the Special Issue}

The first paper, presented by López-Estrada et al. [1], offers an extensive review of the three main topics covered in this special issue. This literature survey presents different methodologies for analysis and control, observer synthesis, and fault-related strategies for convex systems under different representations: Takagi-Sugeno fuzzy models, linear parameter varying (LPV), and quasi-LPV systems.

Zhao et al. [2] perform an analysis on the selection of the length of the control horizon for a linear model predictive control, with application to steam/water loops in large-scale watercraft/ships, with an emphasis on the performance and computational complexity of the algorithm.

Aydın Mühürcü [3] considers a combination of a feed-forward artificial neural network (FFANN) and an artificial bee colony $(\mathrm{ABC})$ optimization algorithm to ensure the settling time of a second-order system. The FFANN is the nonlinear control structure adopted for a buck converter and its parameters are optimized using the $\mathrm{ABC}$ algorithm. 
Gutierrez-Carmona et al. [4] analyze the performance of a nonlinear dissipative observer for a tubular reactor. They show that, by simple considerations in the boundary conditions, the observer's convergence is improved regardless of the presence of perturbations. The sensor locations acquire physical meaning, and by simple numerical manipulations, the inflow perturbations can be estimated numerically.

Son and $\mathrm{Du}[5]$ develop a reliable thermal management system to predict and monitor precisely the thermal behavior of lithium-ion batteries. First, an iterative optimization algorithm corrects the model by incorporating the errors between the measured quantities and the model predictions. Then, an optimization-based fault detection and diagnosis algorithm provide a probabilistic description of the occurrence of possible faults, while taking into account the uncertainties.

Fan et al. [6] present a profile monitoring methodology that includes model fitting and statistical process (SP) control. In this paper, the authors consider non-linear profiles with correlated within-the-profile observations. Three profile models were studied: a traditional one (polynomial regression) but with added autoregression structure, and two known from the theory of non-linear regression, but relatively unknown for SPC practitioners.

Dong et al. [7] present a methodology to assess a specific critical avionic system: the integrated modular avionics (IMA) system. This methodology is derived from a model-based safety analysis performed using the AltaRica 3.0 modeling language. Moreover, the authors present a design optimization of the IMA system.

Zeng et al. [8] present a fault diagnosis and isolation method for gas turbines. First, the measured aerodynamic parameters are decomposed using the kernel principal component analysis. Then, they construct the Hotelling- $T^{2}\left(T^{2}\right)$ statistic, which is the application of the $T$-statistic in multivariate analysis in the principal space and squared prediction error (SPE) statistics in the residual space. Finally, they calculate the parameters' sensitivity to the $T^{2}$ and SPE statistics to locate the fault.

Piprek et al. [9] provide a sampling approach to approximate the chance constraints in the formulation of optimal control problems for stochastic dynamical systems to capture rare events. The applicability of the proposed approach is demonstrated in a battery charging-discharging problem.

Khanum et al. [10] describe an interesting algorithm approach for improving global search minimum optimizations and compare multiple existing algorithms to assess their ability to find optimal parameters for various functions.

Morán-Durán et al. [11] propose the use of a trained neural network to predict and control the voltage of a proton-exchange membrane (PEM) fuel cell. The approach uses principal component analysis (PCA) to reduce the dimensionality, aiming to eliminate non-significant variables with respect to the control objective.

Nguyen et al. [12] present the design of a bilinear model-based predictive control for the three-degrees-of-freedom model of an underactuated ship affected by uncertain disturbances. The bilinear model of the ship is obtained by linearizing each nonlinear model section and the uncertain components and random disturbances of the model are compensated with a state estimator.

Allawi et al. [13] report a novel fine-tuning meta-heuristic algorithm to solve global optimization problems. Also, the proposed algorithm has been validated by comparing it with some featured meta-heuristic optimization algorithms over different benchmark test functions.

Shin et al. [14] discuss a holonic-based mechanism for self-learning factories based on a hybrid-learning approach which is designed to obtain predictive modeling ability in both data-existent and data-absent environments via accommodating machine learning and transfer learning.

Ionescu et al. [15] study the case of an optimization method that considers short-term and long-term cost objectives. The problem of cost-effective optimization of the system's output is studied in a multi-objective predictive control formulation and applied to a windmill park case study.

Zeng et al. [16] provide a method that uses a hybrid filter for fault diagnosis in a gas turbine. The hybrid filter is based on the unscented Kalman filter and a particle filter with optimized weight. 
It estimates the health parameters of the rotor components and builds a model in order to give a prediction for fault diagnosis.

Hernández et al. [17] characterize the high viscosity gas-liquid intermittent flows by detrended fluctuation analysis. Specifically, the authors investigated the long-term evolution of highly viscous two-phase pipe flows of glycerin/air blends. Then they apply a detrended fluctuation analysis of pressure measurements at various positions along the flow line to extract long-range correlations.

Albalawi and Zaid [18] introduce the application of a model-based predictive control algorithm to control and improve the performance of a grid-tied neutral-point-clamped 3- $\varphi$ transformerless inverter powered by a photo-voltaic panel. The controller considers the filter elements, as well as the internal impedance of the grid.

Zheng et al. [19] establish a generalized proportional hazard model to exploit the monitoring condition information of a relay protection equipment to ensure the safe and stable operation of a power system.

Navarro et al. [20] propose a method to detect, locate, and estimate the magnitude of leaks in a pipeline using only flow rate and pressure head measurements at both ends of the pipe. The method develops a mathematical model that builds an observer ensemble using genetic algorithms.

Liu and Lü [21] focus on an approach for fault diagnosis of the blocking diesel particulate filter based on spectral analysis of the instantaneous exhaust pressure. The method is validated experimentally.

Kaid et al. [22] develop a two-step robust deadlock control approach based on Petri nets for automated manufacturing systems where the structural complexity of the Petri net supervisors is minimized.

Cui et al. [23] provide the infrastructure and mathematical tools necessary to face the detection of active distribution networks faults with a wide range of converter interfaces and, therefore, their protection.

Pour et al. [24] present an economic reliability-aware model predictive control based on a finite horizon stochastic optimization problem with joint probabilistic constraints for the management of drinking water transport networks.

Martínez-García et al. [25] propose a discrete-time interval observer for a class of discrete-time parametric uncertain systems modeled in the Takagi-Sugeno form, where the perturbation vector is considered to be unknown but bounded, to estimate state variables and actuator faults.

Tran et al. [26] provide a tuning method for a fuzzy proportional-integral-derivative controller based on a modified genetic algorithm that can speed up convergence and save operation time by neglecting the chromosome decoding step.

Lu et al. [27] study the safety performance of the fly-by-wire system of an aircraft. The safety analysis is based on stochastic simulations of a Simulink model. The Simulink model represents the nominal operation of the system, extended with failure mode. The safety requirements of the system are defined by presenting the thresholds of system performance metrics.

\section{Conclusions}

We believe that the papers in this special issue reveal an exciting area that can be expected to continue to grow in the very near future, namely, the use of advanced optimization strategies in engineering applications. The pursuit of work in this area requires expertise in control engineering as well as in systems design and numerical analysis. We hope that this issue helps to bring these communities into closer contact with each other, as the fruitfulness of collaboration across these areas becomes clear.

Finally, we would like to acknowledge the enthusiastic effort of all the authors, reviewers and editorial staff who have participated in this special issue.

Author Contributions: All authors contributed equally to this work. All authors have read and agreed to the published version of the manuscript. 
Funding: This work was funded by Tecnológico Nacional de México under the program "Investigación Científica Básica y Aplicada". This work has been also supported by the AEI through the Maria de Maeztu Seal of Excellence to IRI (MDM-2016-0656) and the grant Juan de la Cierva-Formacion (FJCI-2016-29019).

Conflicts of Interest: The authors declare no conflict of interest.

\section{References}

1. López-Estrada, F.R.; Rotondo, D.; Valencia-Palomo, G. A Review of Convex Approaches for Control, Observation and Safety of Linear Parameter Varying and Takagi-Sugeno Systems. Processes 2019, 7, 814. [CrossRef]

2. Zhao, S.; Maxim, A.; Liu, S.; De Keyser, R.; Ionescu, C. Effect of Control Horizon in Model Predictive Control for Steam/Water Loop in Large-Scale Ships. Processes 2018, 6, 265. [CrossRef]

3. Mühürcü, A. FFANN Optimization by ABC for Controlling a 2nd Order SISO System's Output with a Desired Settling Time. Processes 2018, 7, 4. [CrossRef]

4. Gutierrez-Carmona, I.; Moreno, J.A.; Abundis-Fong, H. On the Boundary Conditions in a Non-Linear Dissipative Observer for Tubular Reactors. Processes 2018, 7, 8. [CrossRef]

5. Son, J.; Du, Y. Model-Based Stochastic Fault Detection and Diagnosis of Lithium-Ion Batteries. Processes 2019, 7, 38. [CrossRef]

6. Fan, S.K.S.; Jen, C.H.; Lee, J.X. Profile Monitoring for Autocorrelated Reflow Processes with Small Samples. Processes 2019, 7, 104. [CrossRef]

7. Dong, H.; Gu, Q.; Wang, G.; Zhai, Z.; Lu, Y.; Wang, M. Availability Assessment of IMA System Based on Model-Based Safety Analysis Using AltaRica 3.0. Processes 2019, 7, 117. [CrossRef]

8. Zeng, L.; Long, W.; Li, Y. A Novel Method for Gas Turbine Condition Monitoring Based on KPCA and Analysis of Statistics T2 and SPE. Processes 2019, 7, 124. [CrossRef]

9. Piprek, P.; Gros, S.; Holzapfel, F. Rare Event Chance-Constrained Optimal Control Using Polynomial Chaos and Subset Simulation. Processes 2019, 7, 185. [CrossRef]

10. Khanum, R.A.; Jan, M.A.; Tairan, N.; Mashwani, W.K.; Sulaiman, M.; Khan, H.U.; Shah, H. Global Evolution Commended by Localized Search for Unconstrained Single Objective Optimization. Processes 2019, 7, 362. [CrossRef]

11. Morán-Durán, A.; Martínez-Sibaja, A.; Rodríguez-Jarquin, J.P.; Posada-Gómez, R.; González, O.S. PEM Fuel Cell Voltage Neural Control Based on Hydrogen Pressure Regulation. Processes 2019, 7, 434. [CrossRef]

12. Nguyen, H.Q.; Tran, A.D.; Nguyen, T.T. The Bilinear Model Predictive Method-Based Motion Control System of an Underactuated Ship with an Uncertain Model in the Disturbance. Processes 2019, 7, 445. [CrossRef]

13. Allawi, Z.T.; Ibraheem, I.K.; Humaidi, A.J. Fine-Tuning Meta-Heuristic Algorithm for Global Optimization. Processes 2019, 7, 657. [CrossRef]

14. Shin, S.J.; Kim, Y.M.; Meilanitasari, P. A Holonic-Based Self-Learning Mechanism for Energy-Predictive Planning in Machining Processes. Processes 2019, 7, 739. [CrossRef]

15. Ionescu, C.M.; Caruntu, C.F.; Cajo, R.; Ghita, M.; Crevecoeur, G.; Copot, C. Multi-Objective Predictive Control Optimization with Varying Term Objectives: A Wind Farm Case Study. Processes 2019, 7, 778. [CrossRef]

16. Zeng, L.; Dong, S.; Long, W. The Rotating Components Performance Diagnosis of Gas Turbine Based on the Hybrid Filter. Processes 2019, 7, 819. [CrossRef]

17. Hernández, J.; Galaviz, D.F.; Torres, L.; Palacio-Pérez, A.; Rodríguez-Valdés, A.; Guzmán, J.E.V. Evolution of High-Viscosity Gas-Liquid Flows as Viewed Through a Detrended Fluctuation Characterization. Processes 2019, 7, 822. [CrossRef]

18. Albalawi, H.; Zaid, S.A. Performance Improvement of a Grid-Tied Neutral-Point-Clamped 3- $\varphi$ Transformerless Inverter Using Model Predictive Control. Processes 2019, 7, 856. [CrossRef]

19. Zheng, F.; Lin, J.; Huang, J.; Lin, Y. Generalized Proportional Model of Relay Protection Based on Adaptive Homotopy Algorithm Transient Stability. Processes 2019, 7, 899. [CrossRef]

20. Navarro, A.; Delgado-Aguiñaga, J.A.; Sánchez-Torres, J.D.; Begovich, O.; Besançon, G. Evolutionary Observer Ensemble for Leak Diagnosis in Water Pipelines. Processes 2019, 7, 913. [CrossRef]

21. Liu, S.X.; Lü, M. Fault Diagnosis of the Blocking Diesel Particulate Filter Based on Spectral Analysis. Processes 2019, 7, 943. [CrossRef] 
22. Kaid, H.; Al-Ahmari, A.; Li, Z.; Davidrajuh, R. Single Controller-Based Colored Petri Nets for Deadlock Control in Automated Manufacturing Systems. Processes 2019, 8, 21. [CrossRef]

23. Cui, S.; Zeng, P.; Song, C.; Wang, Z. Robust Fault Protection Technique for Low-Voltage Active Distribution Networks Containing High Penetration of Converter-Interfaced Renewable Energy Resources. Processes 2019, 8, 34. [CrossRef]

24. Pour, F.K.; Puig, V.; Cembrano, G. Economic Reliability-Aware MPC-LPV for Operational Management of Flow-Based Water Networks Including Chance-Constraints Programming. Processes 2020, 8, 60. [CrossRef]

25. Martínez-García, C.; Puig, V.; Astorga-Zaragoza, C.M.; Madrigal-Espinosa, G.; Reyes-Reyes, J. Estimation of Actuator and System Faults Via an Unknown Input Interval Observer for Takagi-Sugeno Systems. Processes 2020, 8, 61. [CrossRef]

26. Tran, H.K.; Son, H.H.; Duc, P.V.; Trang, T.T.; Nguyen, H.N. Improved Genetic Algorithm Tuning Controller Design for Autonomous Hovercraft. Processes 2020, 8, 66. [CrossRef]

27. Lu, Z.; Zhuang, L.; Dong, L.; Liang, X. Model-Based Safety Analysis for the Fly-by-Wire System by Using Monte Carlo Simulation. Processes 2020, 8, 90. [CrossRef]

(C) 2020 by the authors. Licensee MDPI, Basel, Switzerland. This article is an open access article distributed under the terms and conditions of the Creative Commons Attribution (CC BY) license (http://creativecommons.org/licenses/by/4.0/). 